\title{
SOME PROPERTIES OF CERTAIN SETS OF COPRIME INTEGERS
}

\author{
ROGER C. ENTRINGER ${ }^{1}$
}

1. Introduction. The set $P(n)$ of all primes less than or equal to $n$ has the obvious property that it contains exactly one multiple of each prime less than or equal to $n$. We use this partial description of $P(n)$ as a basis for the following

DEFINITION 1.1. An increasing sequence $\left\{a_{1}, \cdots, a_{k}\right\}$ of integers greater than 1 is a coprime chain iff it contains exactly one multiple of each prime equal to or less than $a_{k}$.

The following is a list of all coprime chains $\left\{a_{1}, \cdots, a_{k}\right\}$ with $a_{k} \leqq 13$.

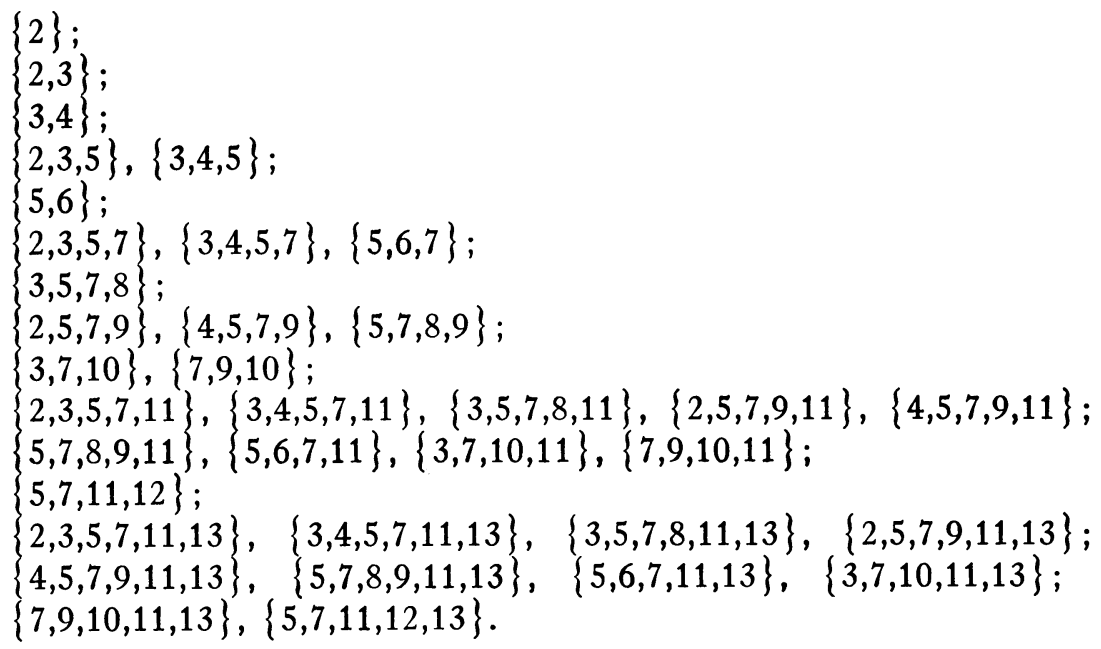

The notation $A(n), B(n)$, etc., will be used to designate coprime chains having $n$ as largest member.

In this paper we are mainly concerned with finding functions asymptotic to the sum of the $r$ th powers of the members of a coprime chain $A(n)$. A later paper will deal with the number of coprime chains with largest member $n$.

All assumed results are well known and can be found in [1].

Received by the editors December 4, 1963.

1 Research supported by the National Science Foundation, Grant G-21219. 
2. The sum of powers of members of a coprime chain. The brief table of coprime chains given in the introduction suggests that a coprime chain $A(n)$ has, asymptotically, $\pi(n)$ members. We will prove a stronger theorem but we first require the

LEMMA 2.1.

$$
\sum_{p \leq n} p^{r} \sim \frac{n^{r+1}}{(r+1) \log n}, \quad r>-1
$$

Proof. We note that for $r=0$ this result is just the prime number theorem. For $r>-1$ partial summation gives

$$
\sum_{p \leq n} p^{r}=\pi(n) n^{r}-r \int_{2}^{n} \pi(t) t^{r-1} d t
$$

But, since

$$
\int_{2}^{n} \pi(t) t^{r-1} d t \sim \int_{2}^{n} t^{r}\left(\frac{1}{\log t}-\frac{1}{(r+1) \log ^{2} t}\right) d t \sim \frac{n^{r+1}}{(r+1) \log n},
$$

we have the desired result.

THEOREM 2.2 .

$$
\sum_{a \in A(n)} a^{r}=\sum_{p \leq n} p^{r}+O\left(\frac{n^{(c r+1) / 2}}{\log n}\right),
$$

where $c=1$ for $-1<r \leqq 0$ and $c=2$ for $r>0$.

Proof. Partition $A(n)$ into the sets $P=\{p \in A(n) \mid p$ is prime $\}$ and $M=A(n)-P$. Then

$$
\sum_{a \in A(n)} a^{r}=\sum_{p \leq n} p^{r}-\sum_{p \leq n ; p \notin P} p^{r}+\sum_{m \in M} m^{r}
$$

Each member of $M$ is divisible by some prime less than or equal to $\sqrt{ } n$ and, since the members of $M$ are coprime in pairs, $M$ has at most $\pi(\sqrt{ } n)$ members.

(i) Assume $-1<r \leqq 0$. For each $m$ in $M$ choose a prime divisor $q$ of $m$. Then

$$
\sum_{m \in M} m^{r} \leqq \sum_{m \in M} q^{r} \leqq \sum_{p \leqq \sqrt{n}} p^{r}=O\left(\frac{n^{(r+1) / 2}}{\log n}\right)
$$

by Lemma 2.1 . 
To complete the proof of this part of the theorem we note that there are at most $\pi(\sqrt{ } n)$ primes $p$ satisfying $\sqrt{ } n<p \leqq n, p \notin P$. Thus

$$
\sum_{p \leq n ; p \notin P} p^{r} \leqq \sum_{p \leq \sqrt{n}} p^{r}+\sum_{\sqrt{n}<p \leq n ; p \notin P} p^{r}=O\left(\frac{n^{(r+1) / 2}}{\log n}\right) .
$$

(ii) Assume $r>0$. Choose $n_{0}>2^{2 / r}$. Then, for $n>n_{0}$,

$$
\sum_{p \leq n} p^{r}-\sum_{p \leq \sqrt{ } n} p^{r}=\sum_{\sqrt{n}<p \leq n} p^{r} \leqq \sum_{a \in A(n)} a^{r} \leqq \sum_{p \leq n} p^{r}+\sum_{m \in M} m^{r} .
$$

Now

$$
\sum_{m \in M} m^{r} \leqq n^{r} \pi(\sqrt{ } n)=O\left(\frac{n^{r+1 / 2}}{\log n}\right)
$$

and

$$
\sum_{p \leq \sqrt{ } n} p^{r}=O\left(\frac{n^{(r+1) / 2}}{\log n}\right), \quad \text { which gives } \sum_{p \leq \sqrt{n}} p^{r}=O\left(\frac{n^{r+1 / 2}}{\log n}\right) .
$$

This completes the proof of the theorem.

Applying Lemma 2.1 to the above result we obtain the

Corollary 2.3 .

$$
\sum_{a \in A(n)} a^{r} \sim \frac{n^{r+1}}{(r+1) \log n}, \quad r>-1 .
$$

As the next theorem shows, Theorem 2.2 is the best possible, in that no error term of lower order will suffice.

THEOREM 2.4. For all sufficiently large $n$, there exist coprime chains $A(n)$ and $B(n)$ such that

$$
\left|\sum_{a \in A(n)} a^{r}-\sum_{b \in B(n)} b^{r}\right| \geqq c_{1} \frac{n^{(c r+1) / 2}}{\log n}
$$

where $c$ is defined as in Theorem 2.2 and $c_{1}$ is a constant depending on $r$ only and is positive for $-1<r \neq 0$.

PRoof. Let $\left\{q_{1}, \cdots, q_{k-1}\right\}$ be the set of primes less than $n$ and not dividing $n$. Let $a_{i}=q_{i}{ }^{\left[\log n / \log q_{i}\right]}, b_{i}=q_{i}, i=1, \cdots, k-1, a_{k}=b_{k}=n$. Then $A(n)=\left\{a_{1}, \cdots, a_{k}\right\}$ and $B=\left\{b_{1}, \cdots, b_{k}\right\}$ are both coprime chains.

(i) Assume $-1<r<0$. Then 


$$
\begin{aligned}
& \left|\sum_{a \in A(n)} a^{r}-\sum_{b \in B(n)} b^{r}\right| \\
& \quad=\sum_{p<n} p^{r}-\sum_{p \mid n} p^{r}-\sum_{p<n} p^{r[\log n / \log p]}+\sum_{p \mid n} p^{r[\log n / \log p]} \\
& \quad \geqq \sum_{p \leqq \sqrt{ } n} p^{r}-\sum_{p \mid n} p^{r}-\sum_{p \leq \sqrt{ } n} p^{r[\log n / \log p]} \\
& \geqq \frac{\{1+o(1)\} n^{(r+1) / 2}}{\frac{r+1}{2} \log n}-\sum_{p \mid n} 1-\sum_{p \leq \sqrt{ } n} p^{r(\log n / \log p-1)} \\
& \geqq \frac{2\{1+o(1)\}}{r+1} \frac{n^{(r+1) / 2}}{\log n}-\sum_{p \leqq \sqrt{ } n} n^{r} p^{-r}=\{1+o(1)\} \frac{-4 r}{1-r^{2}} \frac{n^{(r+1) / 2}}{\log n} .
\end{aligned}
$$

(ii) Assume $r>0$. Then

$$
\begin{gathered}
\left|\sum_{a \in A(n)} a^{r}-\sum_{b \in B(n)} b^{r}\right| \geqq \sum_{p \leqq \sqrt{ } n} p^{r[\log n / \log p]}-\sum_{p \mid n} p^{r[\log n / \log p]}-\sum_{p \leq \sqrt{ } n} p^{r} \\
\geqq \sum_{p \leqq \sqrt{ } n} p^{2 r}-\sum_{p \mid n} n^{r}-O\left(\frac{n^{(r+1) / 2}}{\log n}\right) \\
\geqq\{1+o(1)\} \frac{n^{r+1 / 2}}{\left(r+\frac{1}{2}\right) \log n}+O\left(n^{r} \log n\right)+O\left(n^{(r+1) / 2}\right) \\
=\{1+o(1)\} \frac{2}{2 r+1} \frac{n^{r+1 / 2}}{\log n} .
\end{gathered}
$$

The above theorem is also valid for $r=0$, as will be shown in Theorem 3.5.

The first major difference between coprime chains and sets of consecutive primes becomes apparent in the following

THEOREM 2.5. If, for each $n$, coprime chains $A(n)$ and $B(n)$ are chosen so that $\sum_{a \in \Delta(n)} 1 / a$ and $\sum_{b \in B(n)} 1 / b$ are maximal and minimal, respectively, then

$$
\sum_{a \in A(n)} \frac{1}{a} \sim \log \log n \text { and } \sum_{b \in B(n)} \frac{1}{b} \rightarrow \log 2 \text { as } n \rightarrow \infty .
$$

Proof. Clearly $A(n)-\{n\}$ is the set of primes less than $n$ that do not divide $n$. Hence 


$$
\sum_{a \in A(n)} \frac{1}{a}=\sum_{p \leqq n} \frac{1}{p}-\sum_{p \mid n} \frac{1}{p}+\frac{1}{n}=\log \log n-\sum_{p \mid n} \frac{1}{p}+O(1) .
$$

Since $n$ has no more than $2 \log n$ distinct prime divisors, it follows that

$$
\sum_{p \mid n} \frac{1}{p}=O\left(\log \log \log ^{2} n\right)
$$

for all sufficiently large $n$. Thus

$$
\sum_{a \in A(n)} \frac{1}{a} \sim \log \log n
$$

To complete the proof we now consider any coprime chain $B(n)$ chosen so that $\sum_{b \in B(n)} 1 / b$ is minimal for fixed $n$ and note that $B(n)$ can contain no number less than or equal to $\sqrt{ } n$. We define $P$ and $M$ as in the proof of Theorem 2.2. Then

$$
\begin{aligned}
\sum_{b \in B(n)} \frac{1}{b} & =\sum_{p \in P} \frac{1}{p}+\sum_{m \in M} \frac{1}{m} \\
& =\sum_{\sqrt{ } n<p \leqq n} \frac{1}{p}-\sum_{\sqrt{ } n<p \leqq n ; p \notin P} \frac{1}{p}+O\left(\sum_{m \in M} \frac{1}{\sqrt{ } n}\right) \\
& =\log 2-\sum_{\sqrt{ } n<p \leqq n ; p \notin P} \frac{1}{p}+O\left(\frac{\pi(\sqrt{ } n)}{\sqrt{ } n}\right) \\
& =\log 2-\sum_{\sqrt{ } n<p \leq n ; p \notin P} \frac{1}{p}+o(1) .
\end{aligned}
$$

Again, using the fact that $M$ has at most $\pi(\sqrt{ } n)$ members, we have

$$
\sum_{\sqrt{ } n<p \leq n ; p \notin P} \frac{1}{p}=O\left(\frac{\pi(\sqrt{ } n)}{\sqrt{ } n}\right)=o(1) .
$$

Hence

$$
\sum_{b \in B(n)} \frac{1}{b} \rightarrow \log 2 \text { as } n \rightarrow \infty,
$$

and the proof of the theorem is complete.

If $\{A(n)\}$ is any sequence of coprime chains, then the sequence whose members are $\sum_{a \in A(n)} a^{r}$ is bounded for $r<-1$, but for certain sequences $\{B(n)\}$ we may obtain a more precise result.

ThEOREM 2.6. There exists a sequence $\{B(n)\}$ of coprime chains 
such that the sequence whose members are $\sum_{b \in B(n)} b^{r}$ converges to 0 for all $r<-1$.

Proof. Assume $r<-1,0<\epsilon<1$ given. Choose $n_{0}$ so that $n_{0}^{(r+1) / 2}$ $<\epsilon / \log 2$. Let $\{B(n)\}$ be a sequence of coprime chains chosen so that, for fixed $n, \sum_{b \in B(n)} 1 / b$ is minimal. By Theorem 2.5 there is an $n_{1}$ so that $\sum_{b \in B(n)} 1 / b<\epsilon+\log 2$ for all $n>n_{1}$. Since $B(n)$ contains no number less than or equal to $\sqrt{ } n$ we have $b^{1+r}<n^{(r+1) / 2}$ for all $b$ in $B(n)$. Thus, for all $n>n_{0} n_{1}$, we have $b^{r}<(1 / b) \epsilon / \log 2$ for each $b$ in $B(n)$ and, hence,

$$
0<\sum_{b \in B(n)} b^{r}<\frac{\epsilon}{\log 2} \sum_{b \in B(n)} \frac{1}{b}<\epsilon+\frac{\epsilon^{2}}{\log 2}<3 \epsilon,
$$

and the proof is complete.

3. Coprime chains of maximal and minimal length.

DEFINITION 3.1. For each $n>1$ choose coprime chains $A(n)$ and $B(n)$ so that $\sum_{a \in A(n)} 1$ and $\sum_{b \in B(n)} 1$ are maximal and minimal, respectively. Define

$$
m(n)=\sum_{a \in A(n)} 1 \text { and } l(n)=\sum_{b \in B(n)} 1 .
$$

Now $m(n)$ and $l(n)$ are about the same size; more precisely, setting $r=0$ in Corollary 2.3 gives $m(n) \sim l(n) \sim n / \log n$. However, we can make more precise statements about both $m(n)$ and $l(n)$.

THEOREM 3.2. $l(n)$ assumes every positive integral value.

Proof. From the table in the first section, $l(2)=1$ and, since $l(n)$ $\rightarrow \infty$, it suffices to show $l(n+1) \leqq l(n)+1, n>1$.

Let $\left\{a_{1}, \cdots, a_{k}=n\right\}$ be a coprime chain of minimal length with $a_{i}$, $i=1, \cdots, k-1$, square-free. Let $b_{i}=a_{i} /\left(a_{i}, n+1\right), i=1, \cdots, k-1$. Then the members of $B(n+1)=\left\{b_{1}, \cdots, b_{k-1}, n, n+1\right\}$ are relatively prime in pairs and every prime less than or equal to $n+1 \mathrm{di}$ vides some member of $B(n+1)$. Thus, if all 1's are deleted from $B(n+1)$ and the remaining members are properly reordered, we obtain a coprime chain. Then $l(n+1) \leqq k+1=l(n)+1$ and the proof may be completed by induction.

THEOREM 3.3. $m(n)=\pi(n)-\omega(n)+1$, where $\omega(n)$ is the number of different prime factors of $n$.

Proof. Clearly a coprime chain of maximal length can be constructed by using.only $n$ and all primes less than and relatively prime to $n$. 
COROLlaRy 3.4. $m(n)$ assumes every positive integral value.

Proof. Letting $n=p_{k}$ in the previous theorem we obtain $m\left(p_{k}\right)=k$.

In Theorem 2.4 the restriction $r \neq 0$ is unnecessary in view of the following

ThEOREM 3.5. Given $\epsilon>0, m(n)-l(n)>(1-\epsilon) \sqrt{ } n / \log n$ for all sufficiently large $n$.

Proof. If $\left\{a_{1}, a_{2}, \cdots, a_{2 k-1}, a_{2 k}, a_{2 k+1}, \cdots, n\right\}$ is a coprime chain and $a_{2 k}<\sqrt{ } n$, then $\left\{a_{1} a_{2}, a_{3} a_{4}, \cdots, a_{2 k-1} a_{2 k}, a_{2 k+1}, \cdots, n\right\}$ can be reordered to form a coprime chain. Now the coprime chain containing $n$ as largest member and all primes less than and relatively prime to $n$ contains at least $\pi(\sqrt{ } n)-\omega(n)-1$ members less than $\sqrt{ } n$. By pairing these members as indicated above we can form a coprime chain with at most $m(n)-\frac{1}{2}[\pi(\sqrt{ } n)-\omega(n)-1]$ members. But since $\omega(n)$ $<2 \log n$ we have

$l(n) \leqq m(n)-\frac{1}{2}[\pi(\sqrt{ } n)-2 \log n-1]=m(n)-\frac{\sqrt{ } n}{\log n}\{1+o(1)\}$.

\section{REFERENCE}

1. G. H. Hardy and E. M. Wright, An introduction to the theory of numbers, 3rd ed., Oxford Univ. Press, New York, 1954.

UNIVERSity OF New MeXico 